\title{
Utilization of an Educational Web-Based Mobile App for Acquisition and Transfer of Critical Anatomical Knowledge, Thereby Increasing Classroom and Laboratory Preparedness in Veterinary Students
}

\author{
Kevin Hannon \\ Department of Basic Medical Sciences College of Veterinary Medicine, Purdue
}

\begin{abstract}
Contact time with students is becoming more valuable and must be utilized efficiently. Unfortunately, many students attend anatomy lectures and labs ill-prepared, and this limits efficiency. To address this issue we have created an interactive mobile app designed to facilitate the acquisition and transfer of critical anatomical knowledge in veterinary students, thereby increasing classroom and laboratory preparedness. We have found that in contrast to a traditional reading assignment, utilization of such an app to introduce students to a subject area significantly enhanced the initial learning of anatomy and the transfer of that learned material to a related, but novel area. We propose that students using the apps were subsequently better prepared for lecture and lab, than students using the more traditional method of reading a textbook. Exposure of students to a topic prior to lecture and laboratory, using methods that students embrace, can only lead to a more efficient and better educational experience.
\end{abstract}

Keywords: Mobile educational app; anatomy; class preparedness

Hannon, K. (2017). Utilization of an educational web-based mobile app for acquisition and transfer of critical anatomical knowledge, thereby increasing classroom and laboratory preparedness in veterinary students, Online Learning 21(1), 201-208. doi:

10.24059/olj.v21i1.882

\section{Introduction}

Anatomical instruction is faced with the same challenges of other curriculum: a reduction in contact time while maintaining an equivalent volume of content to be mastered (Sugand, Abrahams, \& Khurana, 2010). Therefore, contact time with students is becoming more valuable and must be utilized efficiently. Unfortunately, many students attend anatomy lectures and labs ill-prepared (“What are we dissecting today?”). This lack of preparedness results in a suboptimal 
use of available contact periods. Current students resist reading traditional texts as pre-lecture or pre-lab assignments, as they find them time consuming and filled with unfamiliar terminology. Therefore, it was our objective to create a web-based learning mobile app with the purpose of preparing students for anatomy lecture and lab. While not used specifically for class preparedness, utilization of web-based computer-aided instructional resources by anatomy students will result in significantly higher scores on examinations (McNulty, Sonntag, \& Sinacore, 2009). Therefore, it is a logical extension to hypothesize that computer-aided instructional resources such as mobile apps will improve anatomy student preparedness. The concept of web-based material for pre-lecture/lab preparation has been introduced previously for a physics curriculum (Novak, 1997). The students' outside-of-class preparation fundamentally affected what happened during the subsequent in-class time together. This type of pre-class preparation also brought a diverse group of students to a similar level before lecture/lab (Novak, 1997; Marrs, \& Novak, 2004). A number of studies have outlined medical students' preference for mobile apps to deliver content as opposed to other more traditional resources (Gutmann, Kühbeck, Berberat, Fischer, Engelhardt, \& Sarikas, 2015; Sandholzer, Rurik, Deutsch, \& Frese, 2014). However fewer have documented the educational effectiveness of mobile web apps. It was the objective of our study to create a web-based anatomy mobile app, and analyze the effectiveness of such an app on the acquisition and transfer of critical anatomical knowledge in veterinary students, thereby increasing classroom and laboratory preparedness.

\section{Method}

The veterinary anatomy app used for this study was web-based and created using a commercial mobile app-creation platform (www.activelessonhq.com). Apps created using this platform are device neutral and therefore could be accessed by students on their computers and/or mobile devices. The utilization of mobile devices is an efficient and popular method to disseminate subject matter to students (Trelease, 2008). The app was designed to be interactive, utilize active retrieval in the form of self-guided quizzes (Karpicke \& Blunt, 2011; Karpicke \& Roediger, 2008), and be simple and intuitive to use. The self-guided quizzes utilized a graphics interface that allowed students to interact with anatomical images on the computer/mobile device monitor. In addition, the answers to the questions with these quizzes could be obtained in two ways: 1) by typing text into an input box, submitting an answer, and getting feedback as to whether the typed answer was correct; and 2) by clicking on a "reveal" button, where the answer was revealed without any text input (Figure 1). To test the efficacy of this app for enhancement of learning of veterinary anatomy, and to examine the importance of the text-input box versus the answer-reveal button, 84 first-year veterinary anatomy students were randomly separated into 3 groups ( $\mathrm{n}=28$ for each group). The age range of this group was 20-37 years old, and there were 67 women and 17 men. Each group was given an equivalent amount of time (2 hrs.) to complete the following tasks: A) Group 1- read a textbook assignment; B) Group 2- Use the anatomy app with text input box, and then read the textbook assignment; and C) Group 3- Use anatomy app with the answer-reveal button, and then read the textbook assignment. The reading assignment and the anatomy app were focused on muscles of the canine scapular region. The reading assignment was in a veterinary anatomy text that had been required for the course for the past 30 years. The app contained interactive images if muscles in situ, isolated muscles and muscle attachments, all information that was included in the reading assignment. The students had no previous exposure to this anatomical area. Upon completion of their assignment, all students were given an identical written exam. The first exam question was a cross-section through the 
canine scapula. The image in this question was found in the textbook reading assignment, but not in the app. Students were asked to identify and write-in the correct muscles (Figure 2). The second exam question was a lateral schematic view of the muscles of the canine scapular region. This image was found in the anatomy app, but not in the reading. Students were again asked to identify and write-in the correct muscles (Figure 3). The third exam question was a lateral schematic view of a dachshund. Students were asked to sketch in specific muscles in the dachshund image (Figure 4). This image was not found in the textbook reading assignment or in the anatomy app. This question tested the student's ability to transfer information they had obtained from the reading assignment and app from a dog with a more "standard" body shape (boxer), to a dog of a more "atypical” body conformation (dachshund). Transfer of anatomical knowledge is a common hurdle that confronts all students of veterinary anatomy, as they dissect and study not only dogs of different body types and breed, but other species (i.e. cat, horse, cow, and pig).

All exams were blind-graded with respect to the assignment group or student identification. Exam questions 1 and 2 were only scored correct if the answers were spelled correctly. Exam question 3 was examined for the correct placement of each attachment for each muscle, and the proper sketching of the muscle between the attachment points. Partial credit was given on question 3. ANOVA and Tukey's honest significance test were used for data analyses. This study was exempted by the Purdue Institutional Review Board.

\section{Results}

As noted above, question 1 asked students to identify canine scapular muscles from a cross-sectional perspective (Figure 2), a task which was covered in the textbook reading, but not in the app. Using the mobile app with either the text-input or reveal-answer function significantly improved scores on Question 1 compared to the read-only group $(F=6.24, p<0.01)$ (Table 1 ). There was no difference between the text-input or reveal-answer groups.

Also as described above, question 2 asked students to identify and write-in the correct names of canine scapular muscles from a lateral perspective (Figure 3), an exercise found the app but not in the reading. Using the mobile app with either the text-input or reveal-answer function significantly improved scores on Question 2 compared to the read-only group $(\mathrm{F}=26.1, \mathrm{p}<0.001)$ (Table 1). There was no difference between the text-input or reveal-answer groups.

In question 3 students were asked to sketch muscles into a lateral view of a dachshund (Figure 4), an image not found in the textbook reading assignment or in the app. Using the mobile app with either the text-input or reveal-answer function significantly improved scores on Question 3 compared to the read-only group ( $\mathrm{F}=40.3, \mathrm{p}<0.001)$ (Table 1, next page). 
Table 1. Exam Question Results.

\begin{tabular}{|l|l|l|l|l|c|c|c|c|c|}
\hline & \multicolumn{4}{|l|}{ Question 1 } & \multicolumn{2}{l|}{ Question 2 } & \multicolumn{2}{l|}{ Question 3 } \\
\hline & $\begin{array}{l}\text { Read } \\
\text { Only }\end{array}$ & $\begin{array}{l}\text { App }+ \\
\text { Text }\end{array}$ & $\begin{array}{l}\text { App }+ \\
\text { Reveal }\end{array}$ & $\begin{array}{c}\text { Read } \\
\text { Only }\end{array}$ & $\begin{array}{c}\text { App }+ \\
\text { Text }\end{array}$ & $\begin{array}{c}\text { App }+ \\
\text { Reveal }\end{array}$ & $\begin{array}{c}\text { Read } \\
\text { Only }\end{array}$ & $\begin{array}{c}\text { App }+ \\
\text { Text }\end{array}$ & $\begin{array}{c}\text { App }+ \\
\text { Reveal }\end{array}$ \\
\hline Range & $0-6$ & $0-6$ & $0-6$ & $0-5$ & $0-7$ & $0-7$ & $0-3.5$ & $0.5-5$ & $1-5$ \\
\hline Average & $1.7^{\mathrm{a}}$ & $3.4^{\mathrm{b}}$ & $2.9^{\mathrm{b}}$ & $1.4^{\mathrm{a}}$ & $4.8^{\mathrm{b}}$ & $4.6^{\mathrm{b}}$ & $1.8^{\mathrm{a}}$ & $3.9^{\mathrm{b}}$ & $4.1^{\mathrm{b}}$ \\
\hline SD & 1.54 & 1.69 & 1.98 & 1.35 & 2.33 & 2.02 & 1.06 & 1.21 & 0.92 \\
\hline SE & 0.29 & 0.31 & 0.37 & 0.26 & 0.44 & 0.38 & 0.20 & 0.22 & 0.17 \\
\hline$p$ & - & $* *$ & $*$ & - & $* * *$ & $* * *$ & - & $* * *$ & $* * *$ \\
\hline
\end{tabular}

Note. Possible ranges were: 0-7 for Questions 1 and 2, and 0-5 for Question 3. Superscripts indicate homogeneous subsets based on Tukey's HSD; $p$ indicates the significance level of the comparison between each intervention group and the Read Only group based on Tukey's HSD: * $p<0.05, * * p<$ $0.01, * * * \mathrm{p}<0.001 . \mathrm{n}=28 /$ group

In summary, using the mobile app significantly improved scores on Questions 1, 2 and 3 when compared to the group that only read the text assignment. Scores within the app groups were significantly higher even on question 1, which contained an image found in the reading and not on the app. Exam scores obtained when using the app with the text input function were not significantly different from when using the app with the reveal answer function. These results demonstrate that the app significantly enhanced the initial learning of anatomy (Questions 1 and 2) and the transfer of that learned material to a related, but novel area (Question 3).

Analysis of the student opinion of the app was analyzed by asking "I felt that the app helped me understand the reading assignment”. The students selected a numerical answer based on a scale spanning from $1=$ yes, to $5=$ no. Students reported feeling that both apps helped in understanding the reading assignment $(\mathrm{App}+$ Text mean $=1.2, \mathrm{SE}=0.13$; $\mathrm{App}+$ Reveal mean $=$ 1.4, SE $=0.13$ ). These results demonstrate that students will accept, utilize and appreciate a mobile learning app when assigned as a precursor to a reading assignment.

\section{Limitations}

One of the limitations of our study design was that we did not perform a pretest of student knowledge of front limb muscle anatomy before they performed the exercises described in this manuscript. We did perform a general anatomy (none of the questions referred to front limb anatomy) pretest on this group of students and found no differences between student groups (data not shown). So the potential exists, albeit highly unlikely, that there was a significant knowledge difference between groups pertaining to front limb muscle anatomy that existed before the experiment. The design of this experiment could have been strengthened by having a pretest and examining and analyzing individual student improvement.

Having mentioned that lack of a pretest might have been a design weakness, it is also important to note that one advantage of our design of not having a pretest is that the students are not clued into "what is important to know" pertaining to the upcoming experiment. 


\section{Discussion}

It was our objective to create an interactive mobile app designed to facilitate the acquisition and transfer of critical anatomical knowledge in veterinary students, thereby increasing classroom and laboratory preparedness. We found that utilization of such an app to introduce students to a subject area significantly enhanced the initial learning of anatomy and the transfer of that learned material to a related, but novel area. We propose that the students using the apps were subsequently better prepared for lecture and lab than the students just reading the textbook assignment. The increased knowledge these students demonstrated on the written exams would clearly help them follow discussion in lecture and facilitate reading a dissection guide and perform dissections. To our surprise, utilization of an app containing a more active form of learning (text input), was not a significantly more effective tool for obtaining anatomical knowledge than an app with a more passive method of learning (answers revealed without input). We hypothesized that using an app with a text input box would be an important consideration when introducing new terminology or when "spelling counts". Regardless, our results support those of McNulty et. al that utilization of web-based computer-aided instructional resources by anatomy students will result in significantly higher scores on examinations (McNulty, Sonntag, \& Sinacore, 2009). In addition as reviewed by Guze, (2015) apps can contribute to educational goals of using technology in medical education include facilitating basic knowledge acquisition. Another positive about mobile applications is student acceptance. Mobile apps are preferred digital learning resources by medical students Gutmann, Kühbeck, Berberat, Fischer, Engelhardt, \& Sarikas, 2015; Sandholzer, Rurik, Deutsch, \& Frese, 2014).

While our study focused on the initial acquisition of anatomical knowledge, our app could still be easily used as a study review tool. The reasons why an app would enhance the initial learning of anatomy could be contributed to many factors, including a simple increase in exposure time to the topic, increased student acceptance when compared to a traditional reading assignment, or by simply being another study option. Studies have shown that successful anatomy students use a multitude of study methods while struggling students relied on single methods (Ward \& Walker 2008). In addition, the apps utilized retrieval practice which has been shown to optimize learning (Karpicke \& Blunt, 2011; Karpicke \& Roediger, 2008). Regardless, exposure of students to a topic prior to lecture and laboratory, using methods that students embrace, can only lead to a better and more efficient educational environment.

\section{References}

Gutmann, J., Kühbeck, F., Berberat, P. O., Fischer, M. R., Engelhardt S, \& Sarikas A. (2015). Use of learning media by undergraduate medical students in pharmacology: a prospective cohort study. PLoS One 10, e0122624.

Guze, P. A. (2015). Using technology to meet the challenges of medical education. Trans Am Clin Climatol Assoc, 126, 260-270.

Karpicke, J. D., \& Blunt, J. R. (2011). Retrieval practice produces more learning than elaborative studying with concept mapping. Science, 331, 772-775. 
Karpicke, J. D., \& Roediger, H. L., 3rd. (2008). The critical importance of retrieval for learning. Science, 319, 966-968.

Marrs, K. A., \& Novak, G. (2004). Just-in-Time teaching in biology: Creating an active learner classroom using the internet. Cell Biology Education, 3, 49-61.

McNulty, J. A., Sonntag, B., \& Sinacore, J. M. (2009). Evaluation of computer-aided instruction in a gross anatomy course: a six-year study. Anat Sci Ed, 2, 2-8.

Novak, E. T. (1997). World wide web technology as a new teaching and learning environment. Int J Mod Phys C, 8, 19-39.

Sugand, K., Abrahams, P., \& Khurana, A. (2010). The anatomy of anatomy: a review for its modernization. Anat Sci Ed, 3, 83-93.

Sandholzer, M., Rurik, I., Deutsch, T., \& Frese T. (2014). Medical students' expectations towards an implementation of a family medicine textbook as a comprehensive app in Germany. J Med Syst, 38, 125-134.

Trelease, R. B. (2008). Diffusion of innovations: smartphones and wireless anatomy learning resources. Anat Sci Ed, 1, 233-239.

Ward P. J., \& Walker J. J. (2008). The influence of study methods and knowledge processing on academic success and long-term recall of anatomy learning by first-year veterinary students. Anat Sci Ed, 1:68-74. 


\section{Figure Legends}

Figure 1. Screen-shot of an example of a quiz question contained within the anatomy app. An answer can be generated by either: 1) typing an answer into the text-input box and receiving automatically graded input on your typed answer by tapping the "Check" button; or 2) by tapping on the "Reveal” button.

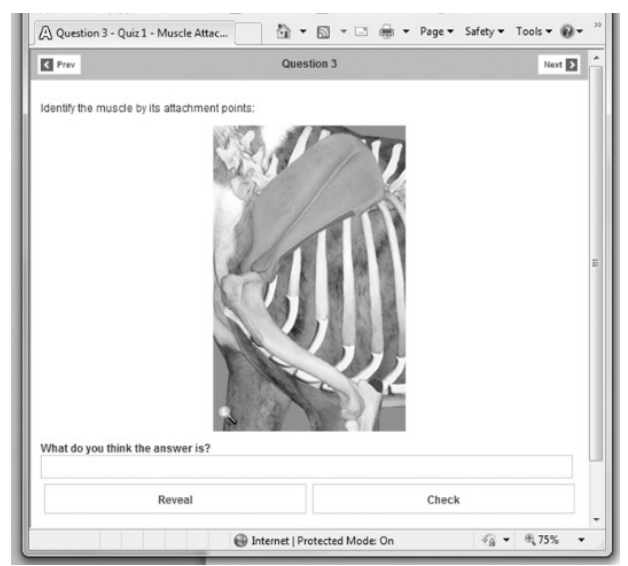

Figure 2. Quiz question 1. The image was found in the traditional reading assignment.

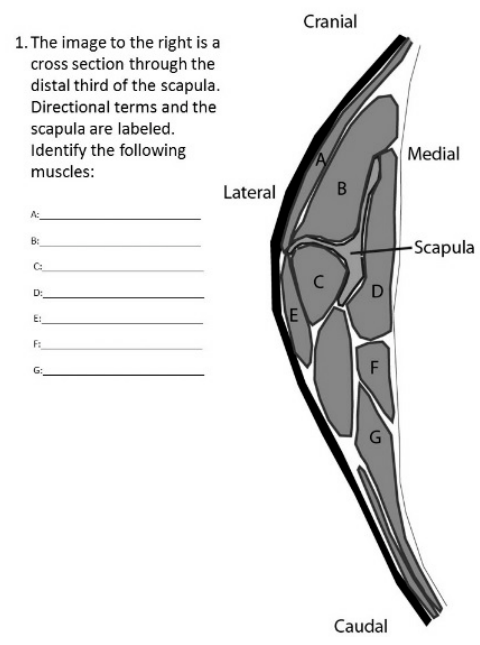


Figure 3. Quiz question 2. The image was found only in the app.
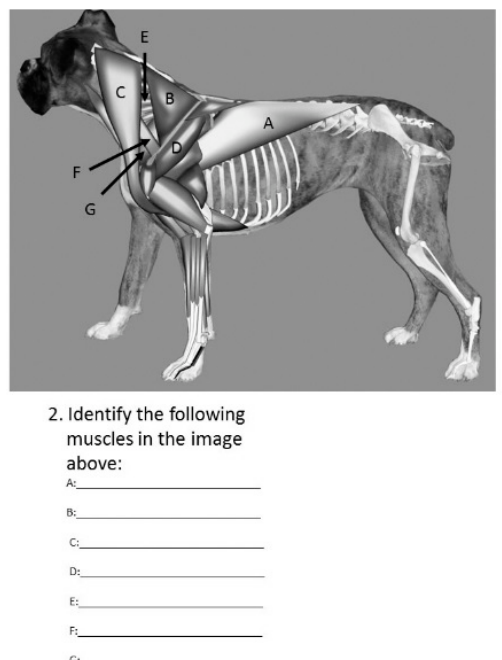

Figure 4. Quiz question 3. The students were not exposed to this image in the reading or the app.

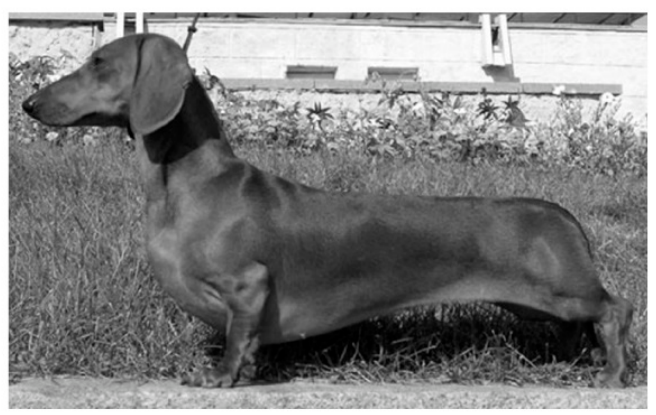

3. Sketch in and label the following muscles on the image above:

a. Rhomboideus

b. Trapezius

c. Omotransversarius

d. Deep pectoral

e. Latissimus dorsi 